The authors identified 1,216 patients who had received at least one DES and who were event-free 6 months after placement. Of these, clopidogrel users had significantly lower incidences of death $(2.0 \%$ vs $5.3 \% ; P=0.03)$ and of a death/Ml composite end point (3.1\% vs $7.2 \% ; P=0.02)$ at 24 months than did nonusers. Furthermore, clopidogrel had a similar effect on 24-month death and death/MI end points in those patients with DESs who were event-free at 12 months. By contrast, clopidogrel use did not predict 24-month outcomes in patients who received BMSs.

The authors suggest that DES placement with long-term (and possibly indefinite) clopidogrel therapy might offer patients the best standard of care, with BMSs an option for those who are unsuitable for extended periods of clopidogrel therapy. They propose a structure for a three-group clinical trial (requiring around 10,000 participants) to assess the optimum duration of clopidogrel therapy in patients who receive a DES.

Original article Eisenstein EL et al. (2007) Clopidogrel use and long-term clinical outcomes after drug-eluting stent implantation. JAMA 297: 159-168

\section{Interactions of inherited and exogenous risk factors increase risk of venous thromboembolism}

Venous thromboembolism (VTE) has many genetic and environmental risk factors, but there are few data on how interactions between these factors contribute to development of the condition. Brouwer et al., therefore, conducted a retrospective analysis of the relative contributions of known hereditary and exogenous risk factors for VTE.

Hereditary defects in protein S, protein C and antithrombin are known to be associated with a high risk of VTE. In this investigation, 1,600 consecutive patients with VTE were screened for deficiencies in these proteins; the screening identified 91 patients with one of these defects, a combined prevalence of $5.7 \%$. Patients and 468 relatives were also tested for plasminogen deficiency, factor $V$ Leiden, excessive levels of factors VIII, IX and XI, hyperhomocysteinemia, and the prothrombin Gly20210Ala polymorphism. Factor $\checkmark$ Leiden was considerably more prevalent in families with protein $\mathrm{C}$ or protein $\mathrm{S}$ deficiency than in the general population; deficiency in protein $\mathrm{C}$, protein $\mathrm{S}$ or antithrombin was associated with increased prevalence of prothrombin Gly20210Ala, high factor VIII levels and hyperhomocysteinemia compared with the general population. In individuals with an index deficiency who had two or more concomitant defects, the annual incidence of VTE was $2.64 \%-103$ times higher than in unaffected individuals (adjusted for clustering in families). Exogenous risk factors increased the risk 2.1-fold.

The authors conclude that VTE results from interactions between multiple inherited and exogenous defects. Families with inherited defects also seem to have a greater prevalence of other risk factors for VTE.

Original article Brouwer JLP et al. (2006) The pathogenesis of venous thromboembolism: evidence for multiple interrelated causes. Ann Intern Med 145: 807-815

\section{Enalapril protects against the cardiotoxic effects of chemotherapy}

High-dose chemotherapy (HDC) can have cardiotoxic effects. Experimental data have indicated that angiotensin-converting-enzyme inhibitors could prevent cardiotoxicity following HDC. Now, Cardinale et al. have shown that angiotensin-converting-enzyme inhibition with enalapril prevents the development of HDC cardiotoxicity, although the mechanism remains to be elucidated.

Increased serum troponin I levels following HDC are a marker for myocardial cell injury and a strong predictor of left ventricular dysfunction; patients who experienced an increase in troponin I levels were, therefore, included in the study. Of 473 consecutive patients who underwent HDC, 114 (24\%) had elevated troponin I levels and were enrolled. Patients were randomly allocated to receive enalapril $(n=56)$ or no additional treatment $(n=58)$. The baseline left ventricular ejection fraction was approximately $62 \%$ in both groups; this figure remained unchanged in the enalapril group, but decreased in the control group to $48.3 \%$ at 12 months. There were 30 cardiac events in the control group, including 14 cases of heart failure and 2 deaths, compared with just 1 event in the enalapril group. Control patients in whom troponin I levels remained elevated 